\title{
ASSOCIAÇÃO ENTRE PERCEPÇÃO DE SAÚDE BUCAL E INDICADORES CLÍNICOS E SUBJETIVOS: ESTUDO EM ADULTOS DE UM GRUPO DE EDUCAÇÃO CONTINUADA DA TERCEIRA IDADE.
}

\author{
ASSOCIATION BETWEEN ORAL HEALTH PERCEPTION AND \\ SUBJECTIVE AND CLINICAL INDEXES: A STUDY OF ADULTS FROM A \\ THIRD AGE CONTINUED EDUCATION GROUP
}

\author{
Dinakel Bortoli ${ }^{1}$, Franciele Aparecida Locatelli ${ }^{2}$, Cristina Berger Fadel ${ }^{2}$, \\ Márcia Helena Baldani \\ 1 Autor para contato: Av. Barão do Rio Branco, 1118 - ap. 503, Marau, RS; \\ e-mail: dinabortoli@yahoo.com.br \\ 2 Universidade Estadual de Ponta Grossa, Campus em Uvaranas, Departamento de \\ Odontologia, Ponta Grossa, PR, Brasil; e-mail: márcia@convoy.com.br
}

Recebido para publicação em 08/09/2003

Aceito para publicação em 25/11/2003

\section{RESUMO}

A odontologia, tradicionalmente, prioriza a utilização de recursos clínicos para diagnosticar as condições de saúde bucal. Esses, entretanto, são limitados, por não informarem o impacto que a condição bucal gera na qualidade de vida dos indivíduos. Este estudo foi realizado para avaliar a autopercepção de saúde bucal em adultos pertencentes ao Grupo de Educação Continuada para a Terceira Idade da UEPG, assim como sua relação com um indicador subjetivo de impactos das condições bucais na qualidade de vida (OHIP-14) e indicadores clínicos (CPO-D e CPI). Foram aplicados dois questinários: autopercepção de saúde bucal e Oral Health Impact Profile (OHIP-14), e os indivíduos submeteram-se a exames clínicos de acordo com a metodologia proposta pela OMS. Os resultados revelaram que 42,1\% dos indivíduos consideraram sua saúde bucal "regular" e 44,7\% a consideraram "boa”. Quanto à percepção de doenças bucais, 39,5\% afirmaram possuir problemas com os dentes e 36,8\% com as gengivas. De acordo com as respostas obtidas através da aplicação do questionário OHIP-14, 60,5\% não apresentaram impacto das condições bucais em sua qualidade de vida. Através da análise de regressão linear simples observou-se uma correlação de $-0,46(p=0,004)$ entre o OHIP-14 e a autopercepção de saúde bucal e de $0,56(p=0,04)$ entre esta e o índice CPI. Não houve correlação significativa entre o OHIP-14 e o índice CPO-D nem entre a autopercepção e esse índice. Através da análise de regressão linear múltipla, evidenciou-se que 52\% da autopercepção de saúde bucal nesse grupo de indivíduos pode ser explicada pelo indicador sub- 
jetivo (OHIP-14) e o de problemas periodontais (CPI).

Palavras-chave: indicadores subjetivos, indicadores clínicos, terceira idade, autopercepção

\begin{abstract}
Tradicional Dentistry prefers to use clinical resources in order to get a diagnosis of oral health status. However, these are considered limited, because they do not inform about the impact the oral health status creates in the population's quality of life. This study was conducted in order to evaluate the self-perception of oral health status in a group of adults who were taking part of the UEPG Third Age Continued Education, in the year 2002, as well as the relation of the perception with a subjective index of impacts of the oral health conditions in the quality of life (OHIP-14) and some tradicional clinical ones (CPO-D and CPI). Two questionaires were applied: a) oral health status self-perception; and b) Oral Health Impact Profile (OHIP-14). The subjects were submitted to clinical examination according to the World Health Organization (WHO). Results revealed that $42.1 \%$ of the members of the group considered their oral health status "regular" and 44.7\% considered it "good". Concerning oral diseases, 39.5\% affirmed that they had problems with their teeth and 36.8\% with their gums. Regarding OHIP-14, 60.5\% didn't present any impact of the oral health status on their quality of life. A simple linear regression analysis pointed at a correlation of $-0,46(\mathrm{p}=0,004)$ between OHIP-14 and self-perception of oral health status, and $-0,56(p=0,04)$ between self-perception and the CPI index. There was not significant correlation between OHIP-14 and the CPO-D index, neither between this index and of the oral health status self-perception. Stepwise procedures of multiple linear regression analysis indicated that almost $52 \%$ of the self-perception of oral health status could be explainded by the subjective index (OHIP-14) and by the periodontal index (CPI).
\end{abstract}

Key words: subjective indicators, clinical indicators, Third Age, selfperception

\section{Introdução}

É real o fato da população mundial estar envelhecendo e isto deve-se, principalmente, ao desenvolvimento de grandes avanços tecnológicos e conseqüente declínio das taxas de mortalidade e fecundidade (Kalache et al., 1987). Essa situação não é diferente na população brasileira, entretanto as prioridades assistenciais em saúde continuam sendo preferencialmente dirigidas às populações infantil e jovem (Veras et al., 1987). A Odontologia, enquanto parte das práticas em saúde, também tem enfrentado essa nova re- alidade, pois tradicionalmente sempre voltou suas atenções a crianças confinadas em instituições de ensino (escolares). Assim sendo, o que se vivencia hoje é um quadro precário de saúde bucal na faixa etária adulta e idosa, resultado da ausência de programas específicos para tal grupo populacional (Silva e Fernandes, 2001).

Frente a esse abandono e dificuldades, ações voltadas à educação em saúde, com ênfase na autopercepção e auto-proteção, deveriam ser mais exploradas (Silva e Fernandes, 2001). Entende-se por educação em saúde quaisquer combinações de experiências de aprendizagem voltadas a facilitar ações 
comportamentais adotadas por uma pessoa, grupo ou comunidade (Candeias, 1997). Na prática, a educação em saúde constitui uma fração das atividades voltadas à melhoria na qualidade de vida e, quando se refere especificamente à saúde bucal, também devem ser priorizadas ações educativas e motivacionais dentro desse contexto. Para que isto seja possível, é preciso buscar uma Odontologia a qual não valorize somente a utilização de recursos clínicos para o diagnóstico das condições de saúde bucal, mas sim, que leve em consideração a forma como a população percebe sua condição de saúde bucal.

Normalmente a avaliação das necessidades populacionais ignora os aspectos sócio-comportamentais e culturais, realizando o planejamento dos serviços de saúde bucal considerando apenas necessidades normativas, pois se enquadra dentro de um modelo biomédico, baseado na identificação de doenças sem levar em consideração a percepção subjetiva do paciente (Leão e Sheiham, 1995).

A literatura oferece vários e diferentes instrumentos que podem ser aplicados com a finalidade da obtenção de dados subjetivos para chegar a um conteúdo válido para a saúde bucal (Atchison e Dolan, 1990; Leão e Scheiham, 1995; Slade e Spencer, 1994; Slade e Spencer, 1997 apud Slade, 1998). Em sua maioria, apresentam-se sob a forma de questionários com questões abertas e fechadas, cuja finalidade básica é o desvendar da autopercepção do paciente no que se refere a sua saúde bucal e como ela interfere no seu dia-a-dia e na sua qualidade de vida. Esses têm se tornando fortes aliados na construção de programas educativos, preventivos e curativos por diversos profissionais da saúde (Silva e Fernandes, 2001).

Em 1994, Slade e Spencer criaram o Oral Health Impact Profile(OHIP), um questionário composto por 49 questões que procura descobrir se o paciente sofreu, nos últimos 12 meses algum incidente social devido à problemas com seus dentes, boca ou próteses. Passados três anos Slade (1997) realizou um estudo com o objetivo de produzir uma versão mais reduzida e menos complexa do OHIP 49, mantendo suas características de confiança e validade. Assim, 14 itens foram selecionados a partir do questionário original, e os mesmos se apresentaram efetivos na detecção das associações clínicas e sócio-demográficas que foram observadas quando foram utilizadas as 49 questões
(Slade, G. D., 1997). O autor chegou à conclusão que o OHIP 14 contém questões que retêm as dimensões conceituais contidas no OHIP 49, e estas questões têm uma boa distribuição de prevalência, sugerindo que o instrumento deva ser utilizado para quantificar níveis de impacto sobre bem-estar, mesmo com um número limitado de questões.

Silva e Fernandes (2001) desenvolveram um estudo com 201 pessoas com idade média de 66,7 anos, funcionalmente independentes, que freqüentavam um centro de saúde localizado em Araraquara-SP, com o objetivo de analisar as associações entre as características sócio demográficas da amostra, a autopercepção da condição bucal e o índice Geriatric Oral Health Assesssment Index (GOHAI). Realizou-se também exame clínico para determinar a prevalência das principais doenças bucais. Os dados subjetivos mostraram que as pessoas apresentaram precária percepção dos problemas bucais. Assim, 60,8\% e 81,3\% dos examinados declararam não ter nenhum problema em seus dentes ou gengiva, respectivamente. A condição bucal foi avaliada como " regular " por $42,7 \%$ das pessoas, e o índice GOHAI apresentou um valor médio de 33,8. As variáveis associadas à auto-avaliação foram: classe social, índice GOHAI, dentes cariados e indicadores para extração. A análise multivariada mostrou que os preditores de auto-avaliação foram o GOHAI, os dentes com extração indicada e o índice Community Periodontal Index and Treatment Needs (CPITN). Esses preditores explicaram 30\% da variabilidade da auto-avaliação. Concluiu-se que a percepção da saúde bucal teve pouca influência nas condições clínicas, mostrando ser necessário desenvolver ações preventivas e educativas para a população.

O presente estudo foi conduzido com o objetivo de avaliar a autopercepção da situação de saúde bucal de adultos pertencentes ao Grupo de Educação Continuada para a Terceira Idade da UEPG, bem como sua relação com um indicador subjetivo de impactos das condições bucais na qualidade de vida e indicadores clínicos tradicionais. Para que a relação pudesse ser avaliada foram utilizados questionários validados na literatura, como o de autopercepção da saúde bucal (Silva e Fernandes, 2001) e o OHIP-14 (Slade, 1997), bem como indicadores clínicos de cárie dentária (CPO-D) e doença periodontal (CPI), como preconizados pela Organização Mundial da Saúde (WHO, 1997). 


\section{Material e método}

Trinta e oito adultos, com idade variando entre 46 e 73 anos, pertencentes ao Grupo de Educação Continuada para a Terceira Idade da UEPG-PR, participaram deste estudo. A coleta dos dados foi realizada entre os meses de abril e novembro do ano de 2002, sendo precedida da assinatura de termo de consentimento livre e esclarecido por parte dos sujeitos da pesquisa.

Para a identificação das necessidades do ponto de vista clínico, todos os participantes do estudo tiveram seus dentes examinados. Foram utilizados, nessa etapa, os índices preconizados pela Organização Mundial da Saúde (WHO, 1997) para a identificação das condições dentais (CPO-D) e periodontais (CPI).

O CPI é o índice destinado às anotações referentes à condição de saúde periodontal, sendo estabelecido em função do sangramento gengival, da presença de cálculos e de bolsas. A dentição é dividida em sextantes, cada qual sendo examinado apenas se possuir dois ou mais dentes presentes e não indicados para extração. O exame é realizado com uma sonda milimetrada específica (sonda CPI, da OMS), em torno de cada dente, com o objetivo de detectar tártaro ou sangramento e determinar a profundidade da bolsa mais profunda. Os códigos variam de 0 a 4, sendo que, 0: corresponde a periodonto hígido; 1: com sangramento; 2: presença de tártaro; 3: bolsa de 4mm à $5 \mathrm{~mm}$; 4: bolsa de $6 \mathrm{~mm}$ ou mais e os sextantes excluídos foram codificados com a letra " $x$ ”. A situação mais grave encontrada no dente é codificada, não devendo o exame ultrapassar, em média, dois minutos. Para o presente estudo, foi considerado o sextante mais afetado de cada indivíduo.

O Î́ndice CPO-D, número médio de dentes permanentes cariados, perdidos e obturados em um grupo de indivíduos, deve ser calculado com base no exame diagnóstico da coroa e da raiz dos dentes presentes em cada indivíduo seguido de anotação em formulário próprio (WHO, 1997). Segundo a OMS, dentes com comprometimento pulpar e com "extração indicada” são computados, nesse formulário, como “cariados”. Na existência de cáries que afetem tanto a coroa quanto a raiz deve ser codificada como cariada apenas a área de origem da lesão (WHO, 1997).

Os exames clínicos foram conduzidos sob condições idênticas: os indivíduos posicionados em cadei- ras escolares em um ambiente com luz artificial e a utilização de materiais e instrumentais clínicos previamente esterilizados (sonda exploradora $n^{\circ} 5$, sonda periodontal CPI, espelho bucal e gaze). Os exames clínicos foram realizados por duas examinadoras, sendo que uma realizou somente o exame periodontal (índice CPI) e a outra registrou o índice de cárie (CPO-D).

Antes da coleta de dados clínicos, procedeu-se à calibração dos examinadores, e verificação da concordância intra-examinador, realizada através do Índice Kappa, Para tal etapa, foi examinada, em dois momentos distintos com intervalo de sete dias, uma população de funcionários da Universidade Estadual de Ponta Grossa, de faixa etária similar àquela do estudo. O exame clínico foi realizado por dois examinadores, sendo que cada um foi calibrado para um dos índices utilizados, que foram o CPO-D e o CPI (Community Periodontal Index ). Os valores de Kappa obtidos foram 0,7 para o CPI e 0,8 para o CPO-D, considerados apropriados segundo Pinto, 2000.

Para a coleta de dados subjetivos foi realizado um estudo piloto, o qual contou com a participação de 6 pessoas pertencentes ao grupo, que posteriormente foram excluídas da amostra. A coleta das informações, no campo subjetivo, foi realizada através da aplicação dos 2 questionários, que foram respondidos individualmente pelos participantes da pesquisa no local de estudo por eles utilizado, em presença dos pesquisadores:

1) um questionário de auto-avaliação da saúde bucal, adaptado de Silva et al. (2001) - Quadro 1. As respostas para a primeira pergunta foram codificadas através de valores de zero até 4 , sendo o valor zero correspondente à percepção de péssima saúde bucal, e o valor 4 correspondente à percepção de saúde bucal excelente.

2) uma versão simplificada do Oral Health Impact Profile, o OHIP-14, conforme preconizado por Slade (1997) - Quadro 2. As respostas foram classificadas de forma dicotômica, sendo que aos itens: "muito freqüente" e "pouco freqüente" foi atribuído valor 1 (um), considerando existência de impacto na qualidade de vida. As respostas "ocasionalmente”, "nunca” e "quase nunca” foram consideradas como inexistência do impacto da condição de saúde bucal na qualidade de vida do indivíduo, recebendo o valor 0 (zero). 
Quadro 1 - Questionário de auto - avaliação da saúde bucal, adaptado de Silva et al., 2001

\section{Auto - avaliação de saúde bucal:}

1 - Como você avalia sua saúde bucal?

( ) excelente ( ) boa ( ) regular ( ) ruim ( ) péssima

2 - Você tem algum problema com seus dentes?

( ) $\operatorname{sim} \quad(\quad)$ não

3 - Você tem algum problema com suas gengivas?

( ) $\operatorname{sim} \quad(\quad)$ não

Quadro 2 - Questionário OHIP - 14 (Slade, 1997)

1 - Você tem dificuldade para pronunciar algumas palavras ou falar devido a problemas com seus dentes, boca ou prótese dentária?

( ) muito freqüente ( ) pouco freqüente ( ) ocasionalmente ( ) quase nunca ( ) nunca

2 - Você sente que seu paladar (sentido do gosto) piorou devido a problemas com seus dentes, boca ou prótese dentária?

( ) muito freqüente ( ) pouco freqüente ( ) ocasionalmente ( ) quase nunca ( ) nunca

3 - Você tem sofrido dores na sua boca ou dentes?

( ) muito freqüente ( ) pouco freqüente ( ) ocasionalmente ( ) quase nunca ( ) nunca

4 - Você sente dificuldade para comer algum alimento devido a problemas com seus dentes, boca ou prótese dentária?

( ) muito freqüente ( ) pouco freqüente ( ) ocasionalmente ( ) quase nunca ( ) nunca

5 - Você se sente inibido por causa de seus dentes, boca ou prótese dentária?

( ) muito freqüente ( ) pouco freqüente ( ) ocasionalmente ( ) quase nunca ( ) nunca

6 - Você tem se sentido tenso por causa de problemas com seus dentes, boca ou prótese dentária?

( ) muito freqüente ( ) pouco freqüente ( ) ocasionalmente ( ) quase nunca ( ) nunca 
7 - Sua dieta tem sido insatisfatória devido a problemas com seus dentes, boca ou prótese dentária?

( ) muito freqüente ( ) pouco freqüente ( ) ocasionalmente ( ) quase nunca ( ) nunca

8 - Você tem interrompido suas refeições devido a problemas com seus dentes, boca ou prótese dentária?

( ) muito freqüente ( ) pouco freqüente ( ) ocasionalmente ( ) quase nunca ( ) nunca

9 - Você sente dificuldade em relaxar devido a problemas com seus dentes, boca ou prótese dentária?

( ) muito freqüente ( ) pouco freqüente ( ) ocasionalmente ( ) quase nunca ( ) nunca

10 - Você tem se sentido embaraçado devido a problemas com seus dentes, boca ou prótese dentária?

( ) muito freqüente ( ) pouco freqüente ( ) ocasionalmente ( ) quase nunca ( ) nunca

11 - Você tem se sentido irritado com outras pessoas devido a problemas com seus dentes, boca ou prótese dentária?

( ) muito freqüente ( ) pouco freqüente ( ) ocasionalmente ( ) quase nunca ( ) nunca

12 - Você tem tido dificuldade de realizar seus trabalhos diários devido a problemas com seus dentes, boca ou prótese dentária?

( ) muito freqüente ( ) pouco freqüente ( ) ocasionalmente ( ) quase nunca ( ) nunca

13 - Você tem sentido a vida menos satisfatória devido a problemas com seus dentes, boca ou prótese dentária?

( ) muito freqüente ( ) pouco freqüente ( ) ocasionalmente ( ) quase nunca ( ) nunca

14 - Você tem se sentido totalmente incapaz de suas obrigações devido a problemas com seus dentes, boca ou prótese dentária?

( ) muito freqüente ( ) pouco freqüente ( ) ocasionalmente ( ) quase nunca ( ) nunca

Os dados foram analisados através do software SPSS 8.0, sendo aplicadas análises de regressão linear simples e múltipla para identificar possíveis associações entre os indicadores utilizados.

\section{Resultados}

A tabela 1 indica que 42,1\% dos indivíduos considerou sua saúde bucal "regular" e 44,7\% a considerou "boa”. Quanto a percepção de doenças bucais, $39,5 \%$ do grupo afirmou possuir problemas com os dentes (tabela 2) e 36,8\% com as gengivas (tabela 3). Com relação ao OHIP 14, 60,5\% da população estudada não apresentou impacto das condições bucais 
em sua qualidade de vida (tabela 4). Observou-se que as pessoas com maior número médio de impactos identificaram uma pior saúde bucal, bem como relataram mais problemas com os dentes e/ou com as gengivas (tabelas 5, 6 e 7).

Os indicadores clínicos demonstraram que o valor médio de CPO-D foi bastante elevado (tabela 8), correspondendo a 22,31 dentes, dos quais 17,81 estiveram classificados na categoria "perdidos”. Com relação o indicador de doença periodontal (não apresentado em tabelas), observou-se que $61,5 \%$ dos indivíduos examinados apresentaram o pior sextante nas categorias 0,1 ou 2, ou seja, com ausência de bolsa periodontal. Os outros apresentaram-se com bolsa periodontal rasa (19,2\%) e bolsa periodontal profunda $(19,2)$ como pior condição clínica periodontal identificada nos sextantes.

Através da análise de regressão linear simples (tabela 9) foi observada uma correlação de -0,46 (p = $0,004)$ entre o OHIP - 14 e a autopercepção de saú- de bucal e de -0,56 ( $p=0,04)$ entre essa e o Índice CPI. Não houve correlação significativa entre o OHIP - 14 e o Índice CPO-D nem entre a autopercepção e esse Índice. Das 14 questões do OHIP, aquelas relacionadas com a dificuldade para comer $(r=-0,50)$, sentir-se inibido ou embaraçado $(r=-0,39)$, interromper as refeições $(r=-0,35)$, e apresentar dificuldades para realizar trabalhos diários $(r=-0,35)$, foram as que mais estiveram correlacionadas com a percepção de melhor ou pior condição bucal (tabela 10).

Através de análise de regressão linear múltipla pelo método stepwise (tabela 11), evidenciou-se um coeficiente de determinação de 0,517 , o que significa que cerca de $52 \%$ da autopercepção de saúde bucal nesse grupo de indivíduos pode ser explicada pelo indicador subjetivo (OHIP - 14) e o de problemas periodontais (CPI).

Os resultados deste estudo podem ser verificados nas tabelas de 1 a 11 .

Tabela 1 - Distribuição da população em estudo segundo critérios de autopercepção da saúde bucal.

\begin{tabular}{ccc}
\hline Critérios & $\mathbf{N}$ & $\mathbf{\%}$ \\
\hline Péssima & 0 & 0 \\
Ruim & 2 & 5,3 \\
Regular & 16 & 42,1 \\
Boa & 17 & 44,7 \\
Excelente & 3 & 7,9 \\
\hline TOTAL & $\mathbf{3 8}$ & $\mathbf{1 0 0}$ \\
\hline
\end{tabular}

Tabela 2 - Distribuição segundo autopercepção de problemas com os dentes.

\begin{tabular}{ccc}
\hline Autopercepção & $\mathbf{N}$ & $\mathbf{\%}$ \\
\hline Não & 23 & 60,5 \\
Sim & 15 & 39,5 \\
\hline TOTAL & $\mathbf{3 8}$ & $\mathbf{1 0 0}$ \\
\hline
\end{tabular}

Tabela 3 - Distribuição segundo autopercepção de problemas com as gengivas.

\begin{tabular}{ccc}
\hline Autopercepção & $\mathbf{N}$ & $\mathbf{\%}$ \\
\hline Não & 24 & 63,2 \\
Sim & 14 & 36,8 \\
\hline TOTAL & $\mathbf{3 8}$ & $\mathbf{1 0 0}$ \\
\hline
\end{tabular}


Tabela 4 - Distribuição segundo o OHIP-14.

\begin{tabular}{ccc}
\hline OHIP $-\mathbf{1 4}$ & $\mathbf{N}$ & $\mathbf{\%}$ \\
\hline 0 & 23 & 60,5 \\
1 & 4 & 10,5 \\
2 & 5 & 13,2 \\
3 & 1 & 2,6 \\
4 & 1 & 2,6 \\
8 & 2 & 5,3 \\
9 & 1 & 2,6 \\
11 & 1 & 2,6 \\
\hline TOTAL & $\mathbf{3 8}$ & $\mathbf{1 0 0}$ \\
\hline
\end{tabular}

* Valor mínimo=0; valor máximo=14

Tabela 5: Média do OHIP-14 para cada critério de autopercepção de saúde bucal.

\begin{tabular}{cccc}
\hline Critérios & N & Média & Desvio padrão \\
\hline Péssima & 0 & 0 & - \\
Ruim & 2 & 9,5 & 2,12 \\
Regular & 16 & 1,19 & 2,07 \\
Boa & 17 & 1 & 2,32 \\
Excelente & 3 & 0,67 & 1,15 \\
\hline TOTAL & $\mathbf{3 8}$ & $\mathbf{1 , 5}$ & $\mathbf{2 , 8 2}$ \\
\hline
\end{tabular}

Tabela 6: Média do OHIP - 14 para autopercepção de problemas com os dentes.

\begin{tabular}{cccc}
\hline Critérios & N & Média & Desvio Padrão \\
\hline Não & 23 & 0,65 & 1,72 \\
Sim & 15 & 2,80 & 3,65 \\
\hline TOTAL & $\mathbf{3 8}$ & $\mathbf{1 , 5 0}$ & $\mathbf{2 , 8 2}$ \\
\hline
\end{tabular}

Tabela 7: Média do OHIP - 14 para autopercepção de problemas com as gengivas.

\begin{tabular}{cccc}
\hline Critérios & N & Média & Desvio Padrão \\
\hline Não & 24 & 0,58 & 1,06 \\
Sim & 14 & 3,07 & 4,05 \\
\hline TOTAL & $\mathbf{3 8}$ & $\mathbf{1 , 5 0}$ & $\mathbf{2 , 8 2}$ \\
\hline
\end{tabular}

Tabela 8: Valor médio de dentes CPO total e por componentes para a população estudada.

\begin{tabular}{ccc} 
Indicador & média & desvio-padrão \\
\hline Hígidos & 5,69 & 4,91 \\
Cariados & 0,04 & 0,20 \\
Perdidos & 17,81 & 8,88 \\
Obturados & 4,46 & 5,08 \\
\hline CPO - D & $\mathbf{2 2 , 3 1}$ & $\mathbf{4 , 9 1}$ \\
\hline
\end{tabular}


Tabela 9: Associação entre autopercepção e os indicadores clínicos e o OHIP-14.

\begin{tabular}{ccc}
\hline Indicadores & $\mathbf{r}$ & $\mathbf{p}$ \\
\hline OHIP -14 & $-0,46$ & $0,004^{*}$ \\
CPI & $-0,56$ & $0,040^{*}$ \\
CPO & $+0,33$ & 0,094 \\
Perdidos & $+0,48$ & $0,014^{*}$ \\
Hígidos & $-0,33$ & 0,094 \\
\hline
\end{tabular}

Tabela 10: Análise de regressão linear simples entre a autopercepção e cada questão do OHIP-14.

\begin{tabular}{ccc}
\hline Questão & $\mathbf{r}$ & $\mathbf{p}$ \\
\hline 1 & $-0,34$ & $0,034^{*}$ \\
2 & $-0,31$ & 0,061 \\
3 & $-0,30$ & 0,070 \\
4 & $-0,50$ & $0,001^{*}$ \\
5 & $-0,39$ & $0,016^{*}$ \\
6 & $-0,23$ & 0,163 \\
7 & $-0,23$ & 0,162 \\
8 & $-0,35$ & $0,030^{*}$ \\
9 & $-0,31$ & 0,059 \\
10 & $-0,35$ & $0,029^{*}$ \\
11 & $-0,33$ & $0,045^{*}$ \\
12 & $-0,35$ & $0,030^{*}$ \\
13 & $-0,29$ & 0,073 \\
14 & $-0,23$ & 0,156 \\
\hline TOTAL & $\mathbf{- 0 , 4 6}$ & $\mathbf{0 , 0 0 4}$ \\
\hline
\end{tabular}

Tabela 11: Resultados da análise de regressão linear múltipla (stepwise) para a autopercepção.

\begin{tabular}{ccccccc}
\hline \hline & \multicolumn{5}{c}{ Coeficiente } \\
\cline { 3 - 6 } $\begin{array}{c}\text { Modelo por ordem de } \\
\text { entrada das variáveis }\end{array}$ & Estimativa & $\begin{array}{c}\text { Erro } \\
\text { padrão }\end{array}$ & p-valor & IC (95\%) \\
\hline \multicolumn{1}{c}{1} & $\mathrm{R}^{2}=0,323$ & & & & & \\
& Constante & 3,973 & 0,170 & $<0,001$ & 3,623 & 4,323 \\
& OHIP-14 & $-0,037$ & 0,011 & 0,002 & $-0,059$ & $-0,014$ \\
\hline 2 & $\mathrm{R}^{2}=0,517$ & 4,372 & 0,197 & $<0,001$ & 3,966 & 4,770 \\
& Constante & $-0,031$ & 0,010 & 0,004 & $-0,051$ & $-0,011$ \\
& OHIP-14 & $-0,238$ & 0,078 & 0,006 & $-0,400$ & $-0,076$ \\
& CPI & & & & & \\
\hline
\end{tabular}

\section{Discussão}

Em busca de um diagnóstico mais amplo e preciso, alguns pesquisadores começaram a trabalhar também com medidas subjetivas, visando inserir a percepção do próprio paciente sobre sua condição bucal como um fator relevante à atenção odontológica (Könönen et al.,1986). Esses dados proporcionam ao profissional, subsídios complementares ao exame clínico, permitindo uma visão integral do paciente e ainda mais dos modernos conceitos de saúde (Silva, 1999). Para a execução de qualquer trabalho específi- 
co dentro da área de saúde é essencial que antes seja traçado um perfil daqueles com quem se está trabalhando, principalmente no caso da geriatria. É de fundamental importância conhecer quais as necessidades e desejos dos indivíduos para que se possa fazer um trabalho efetivo e de segurança.

A falta de informação aos idosos auxiliada pelo descaso dado as suas condições como pacientes especiais, juntamente com a falta de programas para atendimento especializado são os principais indicativos da necessidade de se obter um maior número de pessoas treinadas e capacitadas ao seu atendimento.

Silva (2001) já citava que hoje vivenciamos um quadro precário de saúde bucal da faixa etária adulta e idosa, resultado justamente da ausência de programas específicos para esse grupo populacional e que frente a esse abandono e dificuldades, ações voltadas à educação em saúde, com ênfase na auto-proteção e autopercepção, deveriam ser mais exploradas.

O cirurgião-dentista deveria lançar mão de uma odontologia cujo principal papel não valorize somente a utilização de recursos clínicos para o diagnóstico das condições de saúde bucal; mas sim, que leve em consideração como a população percebe sua própria situação no que se refere aos aspectos de saúde e doenças bucais. Nisso, Leão e Sheiham (1995) já concordavam, dizendo que normalmente a avaliação das necessidades populacionais ignoram os aspectos sócio-comportamentais e culturais, realizando o planejamento dos serviços de saúde bucal levando em consideração uma necessidade normativa, pois se enquadra dentro de um modelo biomédico, baseado na identificação de doenças sem levar em consideração a percepção subjetiva do paciente.

Baseando-se na necessidade de "cuidar" mais das pessoas de terceira idade buscou-se nos indicadores, objetivos em saúde bucal que constituem medidas clínicas úteis para mensuração da necessidade normativa de tratamento do paciente, uma alternativa para melhor atender e compreender a clientela idosa. Entretanto, esses possuem suas limitações, já que não trazem informações a respeito do impacto que a condição bucal possui em sua qualidade de vida.

Os dois questionários aplicados (a) autopercepção de saúde bucal; b) Oral Health Impact Profile - OHIP-14) mostraram a importância que o cirurgião-dentista tem em ouvir seu paciente, sabendo respeitar o que ele acha bom ou ruim para sua qualidade de vida. O segundo questionário utilizado no estudo - OHIP-14 - tem como finalidade básica desvendar a autopercepção do paciente no que se refere a sua saúde bucal e como ela interfere no dia-a-dia e na sua qualidade de vida. Os questionários são fortes aliados na construção de programas educativos, preventivos e curativos por diversos profissionais da saúde (Silva e Fernandes, 2001).

Os indicadores subjetivos devem, ou pelo menos deveriam participar efetivamente do momento da avaliação uma vez que conseguem captar as necessidades relatadas pelos indivíduos e então exibem um panorama mais próximo da sua real condição de saúde bucal. Silva (1999) afirma que tais dados (aspectos bucais clínicos e subjetivos) proporcionam ao profissional subsídios complementares ao exame clínico, permitindo uma visão integral do paciente e ainda mais dos modernos conceitos de saúde.

Os resultados encontrados revelaram que 42,1\% dos indivíduos considerou sua saúde bucal "regular" e 44,7\% a considerou "boa”. Quanto à percepção de doenças bucais, 39,5\% do grupo afirmou possuir problemas com os dentes e 36,8\% com as gengivas. Com relação ao OHIP-14, 60,5\% da população estudada não apresentou impacto das condições bucais em sua qualidade de vida. Através de análise de regressão linear múltipla pelo método stepwise, evidenciou-se que cerca de $52 \%$ da autopercepção de saúde bucal pode ser explicada pelo indicador subjetivo (OHIP-14) e o de problemas periodontais (CPI). Esses resultados foram semelhantes aos obtidos por Silva e Fernandes (2001). Os autores demonstraram em seu estudo que a análise multivariada indicou que os preditores de autoavaliação foram o GOHAI, os dentes com extração indicada e o índice CPITN. Tais indicadores explicaram, neste caso $30 \%$ da variabilidade da auto-avaliação.

A percepção da condição bucal e a importância dada a ela é que condicionam o comportamento do indivíduo. Na maioria das vezes a razão para as pessoas não procurarem o atendimento odontológico é a não percepção de suas necessidades (Silva e Fernandes 2001). Quando as pessoas percebem sua condição bucal, o fazem com uma certa precisão, entretanto, 
usando critérios diferentes dos do profissional. Enquanto o cirurgião dentista avalia a condição com base na ausência ou presença de doença, o paciente dá mais importância aos sintomas e problemas funcionais e sociais que são ocasionados pela presença da doença. No entanto, como constataram Locker e Jokovic (1996), em tempos de escassez de recursos, pode ser muito mais importante identificar subgrupos de pessoas que necessitam de maior atenção do que toda a população. Conseqüentemente, saber se essas medidas são ou não úteis depende dos propósitos e dos objetivos de quem as está utilizando.

\section{Conclusão}

Concluiu-se que os indicadores subjetivos podem ser utilizados como mais um instrumento da avaliação das condições de saúde bucal, complementares aos indicadores clínicos, uma vez que eles conseguem captar as necessidades relatadas pelos indivíduos.

\section{REFERÊNCIAS}

1 ATCHISON, K.A; DOLAN, T.A. Development of the geriatric oral health assessment index. J Dent Educ, v.54, p.680-687, 1990.

2 CANDEIAS, N. M. F. Conceitos de educação e promoção em saúde: mudanças individuais e mudanças organizacionais. Revista Saúde Pública, v.31, n.2, p.209-13, 1997.
3 JOKOVIC, A.; LOCKER, D. Dissatisfaction with oral health status in an older adult population. J Public Health Dent, v.57, p. 40-7, 1997.

4LEÃO A.; SHEIHAM, A. Relationship between clinical dental status and subjective impacts on daily living. J dent Res, v.74, p.1408-1413, 1995.

5 LOCKER, D.; JOKOVIC, A. Using subjective oral health status indicators to screen for dental care needs in older adults. Community Dent Oral Epidemiol, v.24, p.398-402, 1996.

6 PINTO, V.G., Saúde Bucal Coletiva. 4.ed. São Paulo: Santos, 2000.

7 SILVA, S. R. C. Autopercepção das condições bucais em pessoas com 60 anos e mais de idade. São Paulo, 1998. Tese (Doutorado) - Faculdade de Saúde Pública, USP.

8 SILVA, S. R. C.; FERNANDES, R. A. C. Autopercepção das condições de saúde bucal por idosos. Revista Saúde Pública, v.35, n.4, p.01-10, 2001.

9 SLADE, G. D. Assessing change in quality of life using the Oral Health Impact Profile. Community Dent Epidemiol, v.26, p.52-61, 1998.

10 SLADE, G. D. Derivation and validation of a short-form oral health impact profile. Community Dent Oral Epidemiol, v.25, p.284-90, 1997.

11 SLADE, G. D; SPENCER, A. J. Social impact of oral conditions among older adults. Austr Dent J, v.39, p.358-64, 1994.

12 SLADE, G. D.; HOSKIN, G. W.; SPENCER, A. J. Trents and fluctuations in the impact of oral conditions among older adults during a one year period. Community Dent Epidemiol, v.24, p.317-21, 1996.

13 WHO - Oral Health Surveys, Basic Methods. 4.ed. Geneva: World Health Organization, 1997. 